\title{
Occurrence of Polycyclic Aromatic Hydrocarbons in Artisanal Palmero Cheese Smoked with Two Types of Vegetable Matter
}

\author{
M. D. Guillén, ${ }^{1}$ G. Palencia, P. Sopelana, and M. L. Ibargoitia \\ Tecnología de Alimentos, Facultad de Farmacia, Universidad del País Vasco, Paseo de la Universidad, 01006 Vitoria, Spain
}

\begin{abstract}
Palmero cheese is a fresh smoked cheese from the Isle of Palma (Canary Islands), manufactured with goat's milk. To guarantee its safety, the occurrence of polycyclic aromatic hydrocarbons (PAH) in artisanal Palmero cheese smoked with 2 types of vegetable matter (almond shells and dry prickly pear) was studied. The determination of PAH includes extraction and clean-up steps, followed by separation, identification, and quantification of PAH by gas chromatography-mass spectrometry in selected ion-monitoring mode. The most abundant $\mathrm{PAH}$ are those with 2 and 3 aromatic rings. Although the highest total PAH concentrations corresponded to the cheeses smoked with almond shells, the degree of PAH contamination of the cheeses studied was lower than that found in other cheeses smoked in the traditional way. The nature of the vegetable material used for smoking seemed to have an influence on the type of PAH formed, especially on alkylderivatives and some light PAH. However, despite the artisanal, and consequently variable, production process of these cheeses, many similarities have been found among their PAH profiles. In fact, relatively constant relationships are observed between the concentrations of certain pairs of PAH. Benzo(a)pyrene was only present in 2 samples, and in much lower concentrations than the maximum allowed legal limits. Therefore, according to the results obtained, it appears that it is possible to obtain a safe product without renouncing the artisanal character or the sensory properties of this type of cheese.
\end{abstract}

Key words: Palmero smoked cheese, polycyclic aromatic hydrocarbon, gas chromatography-mass spectrometry

\section{INTRODUCTION}

Palmero cheese is an artisanal fresh smoked cheese produced on the Isle of Palma (Canary Islands). This

Received July 18, 2006 .

Accepted January 26, 2007.

${ }^{1}$ Corresponding author: mariadolores.guillen@ehu.es is one of the cheeses protected by a Denomination of Origin (BOE, 2001) and manufactured in the Iberian Peninsula from unpasteurized Palmera goat milk (Freitas and Malcata, 2000). When smoking this kind of cheese, 4 types of vegetable matter can be used: almond shells (Prunus dulcis), dry prickly pear (Opuntia ficus indica), and the wood or needles of the Canary pine (Pinus canariensis).

In addition to conferring special and much appreciated organoleptic characteristics to products, smoking can lead to contamination with polycyclic aromatic hydrocarbons (PAH) if the process is not carefully controlled (Maga, 1988). Polycyclic aromatic hydrocarbons are lipophilic and given the high proportion of fat in cheese, contamination with PAH during the smoking process can occur. However, little research has been done on the occurrence of PAH in this type of food. Moreover, most of the studies focus only on a few PAH (between 5 and 14) (Panalaks, 1976; Joe et al., 1984; Riha et al., 1992; Bosset et al., 1998; De Martin et al., 1998) or exclusively on benzo(a)pyrene (Pagliuca et al., 2003; Anastasio et al., 2004). Nevertheless, despite the limitation of these studies in giving a complete view of the PAH profile of the cheeses studied, all of them reveal the presence of PAH in smoked cheese and, in some cases, in unsmoked cheese.

There is increasing interest in assuring the safety of smoked foods, especially those manufactured following traditional methods. For this reason, and to evaluate the PAH level of Palmero cheese, this paper studied several types of Palmero cheeses produced by different artisans and smoked with 2 of the 4 types of vegetable matter permitted by the Denomination of Origin. Possible relationships between the PAH contamination level of these samples and the degree of smoking were also studied.

\section{MATERIALS AND METHODS}

\section{Samples}

The samples used in this study were 8 smoked Palmero cheeses, manufactured by 8 different artisans. Four of them were smoked with almond shells (AS; i.e, AS2, 
AS4, AS5, and AS6) and the other 4 with dry prickly pear (PP; i.e., PP2, PP3, PP4, and PP5). Previous studies (Guillén and Sopelana, 2004) have shown that most PAH contamination in smoked cheese is concentrated in the outer part. For this reason, and taking into account that the Palmero cheese is fresh and does not have a well-defined rind, the portions studied $(\sim 20 \mathrm{~g})$ were taken from the outer part of the cheeses to a depth of approximately $1 \mathrm{~cm}$. Two aliquots were taken from each cheese.

\section{Reagents}

The solvents used were cyclohexane and methanol, both of HPLC grade (99.9+\%). Other reagents used were potassium hydroxide, sodium chloride, anhydrous sodium sulfate, and sodium tungstate dihydrate. All reagents were commercially available from Aldrich (Steinheim, Germany), Panreac (Barcelona, Spain), and Supelco (Bellefonte, PA).

\section{Standards}

The PAH standards used were the following: a commercial mixture of PAH standards dissolved in a mixture of dichloromethane:benzene (75:25), containing naphthalene, acenaphthene, acenaphthylene, fluorene, phenanthrene, anthracene, fluoranthene, pyrene, benzo(c)phenanthrene, benz(a)anthracene, chrysene, 7,12-dimethylbenz(a)anthracene, benzo(b)fluoranthene, benzo(j)fluoranthene, benzo(k)fluoranthene, benzo(a)pyrene, indeno(1,2,3-cd)pyrene, dibenz(a,h)anthracene, benzo(ghi)perylene, dibenzo(a,l)pyrene, dibenzo(a,i)pyrene and dibenzo(a,h)pyrene at concentrations of approximately $500 \mu \mathrm{g} / \mathrm{mL}$; commercial individual cyclohexane solutions of 1,7-dimethylnaphthalene, 1,4-dimethylnaphthalene, 1,5-dimethylnaphthalene, 1-methylphenanthrene, 2,3dimethylanthracene, 9,10-dimethylphenanthrene, 2methylfluoranthene, 1-methylfluoranthene, $11 \mathrm{H}$ benzo(c)fluorene and 1-methylpyrene at concentrations of approximately $10 \mu \mathrm{g} / \mathrm{mL}$; and a mixture of pure $\mathrm{PAH}$ dissolved in dichloromethane, containing 2,6-dimethylnaphthalene, 2,3-dimethylnaphthalene, o-terphenyl, 2methylanthracene, 9-methylanthracene, 3,6-dimethylphenanthrene, $m$-terphenyl, $p$-terphenyl, $11 \mathrm{H}$-benzo(a)fluorene, $11 \mathrm{H}$-benzo(b)fluorene, benzo(e)pyrene and perylene, in concentrations ranging from 100 to 247.5 $\mu \mathrm{g} / \mathrm{mL}$.

Naphthalene- $\mathrm{d}_{8}$, acenaphthene- $\mathrm{d}_{10}$, phenanthrene$\mathrm{d}_{10}$, pyrene- $\mathrm{d}_{10}, p$-terphenyl- $\mathrm{d}_{14}$, chrysene- $\mathrm{d}_{12}$, perylene$\mathrm{d}_{12}$, and benzo(ghi)perylene- $\mathrm{d}_{12}$ were used as internal standards. The purity of all standards ranged from 97 to $99.5 \%$.
All the above-mentioned standards were used in the identification and quantification of the $\mathrm{PAH}$ present in the samples. All pure standards and solutions were obtained from Sigma, Aldrich, Supelco, and Symta (Madrid, Spain).

\section{Determination of PAH}

The methodology used for the study of the samples was described elsewhere (Guillén and Sopelana, 2004). In brief, the method includes the addition of a mixture of deuterated internal standards to the ground cheese, extraction of fat with cyclohexane in an ultrasonic bath, filtration, alkaline treatment of the fat with boiling methanolic potassium hydroxide for $4 \mathrm{~h}$ under reflux, extraction of PAH by shaking with cyclohexane in a separator funnel, washing of the extract, clarification with sodium tungstate, filtration, drying with anhydrous sodium sulfate, and purification with solid-phase extraction tubes filled with silica [Supelclean LC-Si SPE tubes $3 \mathrm{~mL}$ (500 mg); Supelco]. The final extract was studied by gas chromatography-mass spectrometry in selected ion-monitoring (SIM) mode. This was carried out by means of a Hewlett-Packard gas chromatograph (model HP 6890 Series) equipped with a mass selective detector (model 5973) and a Hewlett-Packard Vectra XM Series 4 computer (Hewlett-Packard, Palo Alto, CA. The column used was a fused-silica capillary column (60 m long $\times 0.25 \mathrm{~mm}$ inner diameter $\times 0.25 \mu \mathrm{m}$ film thickness), coated with a nonpolar stationary phase (HP-5MS, 5\% phenyl methyl siloxane). The operating conditions were as follows: the oven temperature was set initially at $50^{\circ} \mathrm{C}$ (followed by a 0.50 -min hold), increased to $130^{\circ} \mathrm{C}$ at $8^{\circ} \mathrm{C} / \mathrm{min}$, and increased to $290^{\circ} \mathrm{C}$ (followed by a 70 -min hold) at a rate of $5^{\circ} \mathrm{C} / \mathrm{min}$; the temperatures of the ion source and the quadrupole mass analyzer were kept at 230 and $150^{\circ} \mathrm{C}$, respectively. Helium, with a purity of $99.999 \%$, was used as carrier gas at a constant flow of $1.0 \mathrm{~mL} / \mathrm{min}$; injector and transference line temperatures were held at 280 and $300^{\circ} \mathrm{C}$, respectively; pulsed splitless mode was used for injection with a pressure pulse of $206.84 \mathrm{kPa}$, and $1 \mu \mathrm{L}$ of each sample was introduced into the gas chromatograph. Scan mode was occasionally used to ascertain the type of compounds present in the samples.

Identification of the compounds was based on their retention times and on the relative abundances of the ions selected for their identification. Quantification in selected ion monitoring mode is based on the measurement of the peak area corresponding to the most abundant ion of each compound, and was carried out by means of the deuterated internal standards previously mentioned. Thus, naphthalene- $\mathrm{d}_{8}$ was used for quantification of naphthalene and its alkylderivatives; acen- 
aphthene- $\mathrm{d}_{10}$ for acenaphthylene and acenaphthene; phenanthrene- $\mathrm{d}_{10}$ for fluorene, phenanthrene, anthracene, and their alkylderivatives; pyrene- $\mathrm{d}_{10}$ for fluoranthene and pyrene; $p$-terphenyl- $\mathrm{d}_{14}$ for $m$-terphenyl, $p$-terphenyl, benzofluorenes, and methyl-fluoranthenes and methyl-pyrenes; chrysene- $\mathrm{d}_{12}$ for benz(a)anthracene and chrysene; perylene- $\mathrm{d}_{12}$ for benzofluoranthenes and benzopyrenes; and benzo(ghi)perylene- $\mathrm{d}_{12}$ for PAH with higher molecular weights.

\section{Semiquantification of Headspace Volatile Components}

Analysis of headspace volatile components was accomplished by using solid-phase microextraction (SPME) for isolation of the headspace components and GC-MS for separation, identification, and semiquantification as described previously (Guillén et al., 2004a,b).

\section{Statistical Analysis}

Student's $t$-test analysis of independent samples was used to compare the results from the 2 groups of cheeses with regard to the smoking material. The statistical analysis was performed using the SPSS v.14.0 software package for Windows (SPSS Inc., Chicago, IL).

\section{RESULTS AND DISCUSSION}

The compounds detected and their concentrations in the cheeses smoked with almond shells and with dry prickly pear are shown in Table 1 . Results are given as the mean value of the results corresponding to 4 cheeses per vegetable matter, considering 2 aliquots per cheese ( \pm standard deviation), together with the range of concentrations observed. As expected, the results revealed that the outer parts of all the cheeses studied contain $\mathrm{PAH}$, even though the total PAH concentrations were much lower in the samples smoked with dry prickly pear $(29.21$ to $193.11 \mu \mathrm{g} / \mathrm{kg}$ ) compared with those smoked with almond shells (42.46 to $739.55 \mu \mathrm{g} / \mathrm{kg}$ ).

The presence of very light (naphthalene or acenaphthylene) and heavy PAH (benz(a)anthracene, chrysene and even of benzopyrenes or benzo(ghi)perylene in some samples, such as AS5 and PP3) is worth noting (Table 1). Naphthalene and its alkylderivatives are the main $\mathrm{PAH}$ present in these cheese samples as well as in commercial smoke flavorings (Guillén et al., 2000a), smoke flavorings generated from different kinds of wood (Guillén et al., 2000b), smoke produced from several vegetable matters (Conde et al., 2005), and commercial smoked cheeses (Guillén and Sopelana, 2004). As reported in the above studies, both the number and the concentration of light $\mathrm{PAH}$ are much greater than those of heavier PAH. In general, as the molecular weight of the compounds increases, their concentration decreases, such that the samples having more light PAH (AS5 and PP3) were richer in heavier PAH, too.

In agreement with the above-mentioned studies, particularly noteworthy is the high number of alkylated $\mathrm{PAH}$, which, in this work, account for a proportion of the total PAH concentration ranging from 60.5 to $67.0 \%$, even though this group of $\mathrm{PAH}$ are rarely considered in the studies related to smoked cheese or to food in general. They are mainly derivatives of naphthalene, but some are derived from phenanthrene, fluoranthene, or pyrene.

It is worth pointing out that although each sample was manufactured by a different artisan and presents a different degree of PAH contamination, some common features can be observed in the PAH profiles. Table 2 shows the ratios between the concentrations of some pairs of isomers in all the samples studied, together with their total PAH content; certain regularity is observed in these data. With respect to unsubstituted or parent $\mathrm{PAH}$, it should be noticed that there is a very close relationship between the concentrations of some pairs of isomers. Thus, as can be seen, phenanthrene is always present at a greater concentration than anthracene, although the proportion between these $2 \mathrm{PAH}$ seems to vary depending on their concentration level; in fact, the most contaminated cheese samples (AS5 and PP3) showed the lowest values for this ratio. In a similar way, the sum of chrysene and triphenylene is always greater than the concentration of ben$z$ (a)anthracene.

The most notable pair of isomers was fluoranthene and pyrene, whose concentrations almost always maintained the same proportion, between 1.2 and 1.5, independently of their concentration level (fluoranthene being slightly more abundant). Similar ratios were observed in the studies mentioned above on the occurrence of PAH in smoke flavorings (Guillén et al., 2000a,b), smoke (Conde et al., 2005), and commercial smoked cheeses (Guillén and Sopelana, 2004). It is worth noting that when the concentrations of fluoranthene and pyrene are lower than $1 \mu \mathrm{g} / \mathrm{kg}$, benzofluoranthenes, benzopyrenes, or $\mathrm{PAH}$ with greater molecular weights are not detected.

There is also a similar trend in the concentrations of alkylated PAH (Table 2), as can be seen for the 2 monomethylnaphthalenes; the ratio of their concentrations ranged between 1.2 and 1.6. With regard to dimethylnaphthalenes, it can be observed (Table 1) that, in general, their concentrations are lower than those of monomethylderivatives. Whereas the levels of 2,6-, 1,7-, and 1,6-dimethylnaphthalenes are very similar, the rest of the isomers are present at lower concentra- 
Table 1. Polycyclic aromatic hydrocarbons (PAH) identified in the outer part of the cheeses smoked using almond sheels or dry prickly pear and their concentrations $(\mu \mathrm{g} / \mathrm{kg})^{1}$

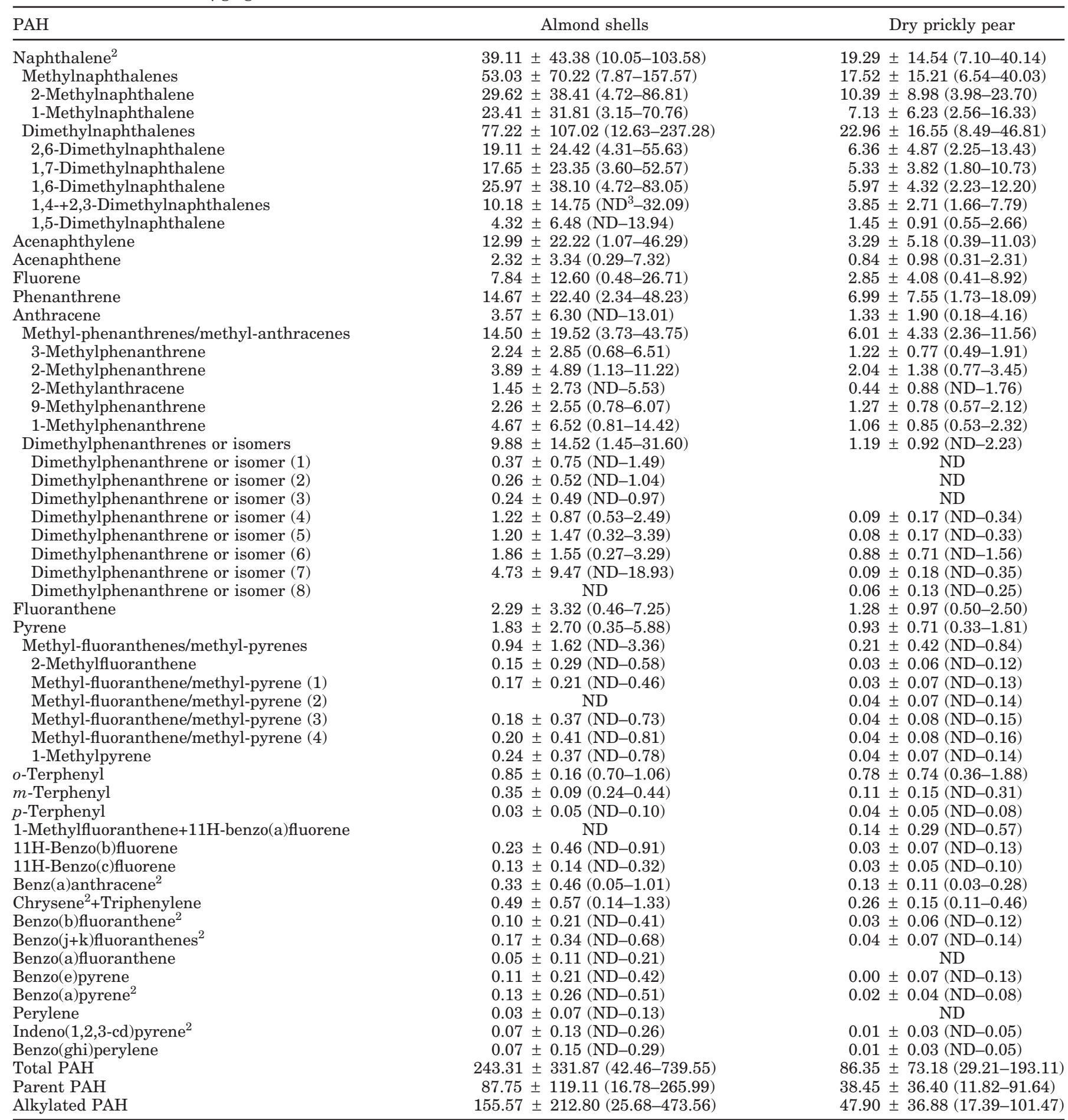

\footnotetext{
${ }^{1}$ Results are given as the mean value of 4 cheeses per each vegetable matter, considering 2 aliquots per cheese $( \pm$ SD) together with the
} range of concentrations observed.

${ }^{2} \mathrm{PAH}$ with a certain degree of carcinogenicity according to the classification of the International Agency for Research on Cancer (IARC, 1983, 2002; Straif et al., 2005).

${ }^{3} \mathrm{ND}=$ not detected. 
Table 2. Ratios between the concentrations of some pairs of isomers in the different cheeses studied, together with their total polycyclic aromatic hydrocarbon $(\mathrm{PAH})$ content

\begin{tabular}{|c|c|c|c|c|c|c|c|}
\hline \multirow[b]{2}{*}{ Cheese samples ${ }^{1}$} & \multicolumn{6}{|c|}{ Pairs of isomers ${ }^{2}$} & \multirow{2}{*}{$\begin{array}{c}\text { PAH content, } \\
\mu \mathrm{g} / \mathrm{kg} \text { of } \\
\text { outer part }\end{array}$} \\
\hline & $\mathrm{Ph}: \mathrm{A}$ & Ft:P & $\mathrm{C}+\mathrm{T}: \mathrm{BaA}$ & BFts:BPs & Bper:IP & 2-MN:1-MN & \\
\hline \multicolumn{8}{|l|}{ Almond shells } \\
\hline AS5 & 3.7 & 1.2 & 1.3 & 1.4 & 1.1 & 1.2 & 739.55 \\
\hline AS6 & 6.2 & 1.3 & 3.0 & - & - & 1.3 & 105.52 \\
\hline AS4 & 6.6 & 1.2 & 1.8 & - & - & 1.5 & 85.71 \\
\hline AS2 & - & 1.4 & 2.8 & - & - & 1.5 & 42.46 \\
\hline \multicolumn{8}{|l|}{ Dry prickly pear } \\
\hline PP3 & 4.3 & 1.4 & 1.6 & 1.2 & 1.0 & 1.5 & 193.11 \\
\hline PP5 & 8.9 & 1.3 & 1.7 & - & - & 1.4 & 70.75 \\
\hline PP2 & 7.6 & 1.4 & 3.7 & - & - & 1.5 & 52.31 \\
\hline PP4 & 9.6 & 1.5 & 3.0 & - & - & 1.6 & 29.21 \\
\hline
\end{tabular}

${ }^{1}$ Samples were smoked using either almond shells or dry prickly pear.

${ }^{2} \mathrm{Ph}=$ phenanthrene; $\mathrm{A}=$ anthracene; $\mathrm{Ft}=$ fluoranthene; $\mathrm{P}=$ pyrene; $\mathrm{C}+\mathrm{T}=$ chrysene + triphenylene; $\mathrm{BaA}=$ benz(a)anthracene; BFts = benzo(b)fluoranthene + benzo(j)fluoranthene + benzo(k)fluoranthene + benzo(a)fluoranthene; BPs = benzo(e)pyrene + benzo(a)pyrene; Bper = benzo(ghi)perylene; IP = indeno(1,2,3cd)pyrene; $2-\mathrm{MN}=2$-methylnaphthalene; $1-\mathrm{MN}$ = 1-methylnaphthalene.

tions, especially 1,5-dimethylnaphthalene. It can also be observed that the sum of the concentrations of monomethylnaphthalenes is of a similar order to the concentration of naphthalene in most of the samples; in contrast, the total of dimethylnaphthalenes is always greater than the amount of naphthalene.

Considering the alkylderivatives of phenanthrene and anthracene, we observed (Table 1) that the concentrations of 3- and 9-methylphenanthrenes are very similar, and that the level of 2-methylanthracene is always lower than that of the methylphenanthrenes, in accordance with the concentration of their corresponding parent PAH. The total concentration of monoalkylated derivatives is of a similar order to the concentration of phenanthrene, and both are higher than the sum of dialkylated derivatives.

Finally, the individual concentrations of methyl-fluoranthenes and methyl-pyrenes and their sum are always lower than the concentrations of their parent PAH.

If the results obtained in the current study are compared with those of other authors, it must be taken into account that the number of PAH determined is always lower than in this work. The levels reported in the current study are lower than those found in other Spanish commercial cheeses smoked in the traditional way, where total PAH concentrations ranged from 36.31 to $1,037.23 \mu \mathrm{g} / \mathrm{kg}$ (Guillén and Sopelana, 2004). These results confirm that if the smoking process is controlled it is possible to reduce the PAH levels of smoked cheese and obtain a safe product without sacrificing the artisanal character or the sensory properties of the product.

To evaluate if there was a relationship between the type of vegetable matter used in the smoking process and the PAH profile of the smoked cheeses, the ratios between the concentrations of certain PAH were calculated and are shown in Table 3. The first 4 ratios referenced relationships between some $\mathrm{PAH}$ and their alkylated derivatives (alkylation ratios), whereas the others (ratios 5 to 8) concern pairs of PAH that differ in one aromatic ring (aromaticity ratios). It can be observed from this table that the values corresponding to the alkylation ratios are somewhat higher in the cheeses smoked with dry prickly pear than in those smoked with almond shells, especially in the case of ratio 4 (phenanthrene/dimethylphenanthrenes). This suggested that the formation of alkylated PAH during the process of smoke generation, particularly of dimethylphenanthrenes, was more favored when almond shells were used. Otherwise, the values of the alkylation ratios reflected those given in Table 1 with regard to alkylated derivatives.

As noted earlier, Table 3 shows some ratios between pairs of PAH that differ in one aromatic ring (ratios 5 to 8). These PAH were selected taking into account one of the mechanisms proposed for the formation of $\mathrm{PAH}$ during the pyrolysis of wood. According to this mechanism, during combustion at high temperatures and with relatively low oxygen content, organic matter breaks down leading to the formation of smaller fragments, most of them free radicals, that recombine to form PAH as the temperature increases. Thus, the successive recombination of radicals such as acetylene $\left(\mathrm{C}_{2}\right)$ and 1,3-butadiene $\left(\mathrm{C}_{4}\right)$ results in the formation of nonsubstituted PAH (Badger et al., 1960; Crittenden and Long, 1976), and once the lightest PAH have been formed, heavier PAH can be formed from the latter PAH by addition of more small units to aryl radicals (Frenklach and Warnatz, 1987). In fact, the formation of phenanthrene from naphthalene and of benzo(a)pyr- 
Table 3. Ratios between the concentrations of some pairs of polycyclic aromatic hydrocarbons in the different cheeses studied

\begin{tabular}{lcccccccr}
\hline & \multicolumn{7}{c}{ Cheese samples $^{2}$} \\
\cline { 2 - 8 } Ratio $^{1}$ & AS5 & AS6 & AS4 & AS2 & PP3 & PP5 & PP2 & PP4 \\
\hline Alkylation ratios & & & & & & & & \\
1. N/MNs & 0.7 & 0.8 & 1.0 & 1.3 & 1.0 & 1.4 & 1.1 & 1.1 \\
2. N/DMNs & 0.4 & 0.8 & 0.7 & 0.8 & 0.9 & 1.0 & 0.7 & 0.8 \\
3. Ph/MPhs & 1.1 & 0.6 & 0.9 & 0.6 & 1.6 & 0.7 & 1.0 & 0.7 \\
4. Ph/DMPhs & 1.5 & 0.8 & 1.9 & 1.6 & 8.1 & 4.0 & 2.4 & - \\
Aromaticity ratios & 2.2 & 16.4 & 5.9 & 9.4 & 3.6 & 14.1 & 31.8 & 14.8 \\
5. N/Al & 2.1 & 7.1 & 4.0 & 4.3 & 2.2 & 3.3 & 4.4 & 4.1 \\
6. N/Ph & 8.2 & 9.8 & 6.4 & 6.3 & 10.0 & 4.4 & 7.6 & 5.2 \\
7. Ph/Pyr & 11.5 & - & - & - & 22.6 & - & - & - \\
8. Pyr/B(a)pyr & & & & & & & &
\end{tabular}

${ }^{1} \mathrm{~N}=$ naphthalene; $\mathrm{MNs}$ = methylnaphthalenes; $\mathrm{DMNs}$ = dimethylnaphthalenes; $\mathrm{Ph}$ = phenanthrene; $\mathrm{MPhs}=$ methylphenanthrenes; DMPhs = dimethylphenanthrenes; $\mathrm{Al}=$ acenaphthylene; $\mathrm{Pyr}=$ pyrene; $\mathrm{B}(\mathrm{a})$ pyr $=$ benzo(a)pyrene.

${ }^{2}$ Cheese samples were smoked using either almond shells (AS) or dry prickly pear (PP).

ene from pyrene have been observed previously (Möhler, 1980).

It can be observed from Table 3 that in the most contaminated samples (AS5 and PP3), the values of ratios 5 and 6 are lower than in the rest of the samples smoked with each type of vegetable matter. This could suggest that the smoke generation and smoking conditions that give rise to higher concentrations of PAH also result in higher proportions of acenaphthylene and phenanthrene in relation to naphthalene. On the other hand, the lower values of ratio 6 in relation to ratio 5 suggest more formation of phenanthrene against acenaphthylene with both vegetable matters. Finally, the values of ratio 5 in the cheeses smoked with almond shells are lower than in the cheeses smoked with dry prickly pear, revealing a greater trend to form acenaphthylene in the pyrolysis process of almond shells.

In connection with ratio 7 , which would express the formation of pyrene from phenanthrene, the values are in the same range in the cheeses smoked with almond shells (6.3 to 9.8$)$ as in those smoked with dry prickly pear (4.4 to 10.0). Finally, the value of ratio 8 in sample AS5 (11.5) is lower than in sample PP3 (22.6), showing a higher proportion of benzo(a)pyrene in the cheese smoked with almond shells.

It can be concluded that, in spite of the differences in the smoking processes applied by different artisans and in the vegetable matter used for smoke generation, the PAH profile of all cheeses studied could be considered quite similar. In fact, there were some relationships between certain $\mathrm{PAH}$, such as fluoranthene and pyrene, that remained constant from one sample to another, irrespective of the conditions of the smoking process; this could be useful predicting or estimating the levels of some PAH from others. Nevertheless, the relationship between pairs of $\mathrm{PAH}$ such as phenan- threne and anthracene, naphthalene and acenaphthylene, or naphthalene and phenanthrene seemed to be influenced by their concentration level.

Despite the similarities found among all the samples studied, there were some differences between the PAH profiles of the cheeses smoked with almond shells and with dry prickly pear. Thus, dimethylderivatives of both naphthalene and phenanthrene were slightly more abundant in the cheeses smoked with almond shells. These differences in the content of alkylated PAH implied that, during the pyrolization of dry prickly pear, alkylderivatives are produced to a lesser extent than in the pyrolization of almond shells, influencing the proportion of these compounds in the smoked cheeses. Under the same combustion conditions, some woods produce smoke with higher concentrations of alkylated PAH than others (Guillén et al., 2000b). Thus, the nature of the vegetable matter used for smoking appears to affect the formation of some PAH and influence the $\mathrm{PAH}$ profile obtained, especially on those compounds of low molecular weight.

To determine if the apparent differences observed between the 2 groups of cheeses were statistically significant, a Student's $t$-test analysis was used to compare the values of ratios 1 to 7 from Table 3 . The results obtained did not show any significant differences between groups of samples for any of the ratios considered, probably due to the high variability existing among the cheeses smoked with the same vegetable matter but produced by different artisans. This variability in the PAH concentration level of the cheeses studied could be attributed to the smoking conditions applied by each artisan. The factors that determine the PAH level in smoked foods are numerous; these include the vegetable matter used to produce the smoke and all the parameters involved in the pyrolysis process, 
Table 4. Concentrations of some groups of typical smoke components in the headspace of the cheeses studied, together with the concentrations of heavy, light, and total polycyclic aromatic hydrocarbons $(\mathrm{PAH})$

\begin{tabular}{|c|c|c|c|c|c|c|c|c|}
\hline \multirow[b]{3}{*}{ Sample $^{1}$} & \multicolumn{5}{|c|}{ Smoke components, area counts $/ 10^{5}$} & & & \\
\hline & \multirow{2}{*}{$\frac{\text { Carb. der. }^{2}}{\text { (Group 1) }}$} & \multicolumn{3}{|c|}{ Phenolic derivatives $^{3}$} & \multirow[b]{2}{*}{ Total } & \multicolumn{3}{|c|}{$\mathrm{PAH},{ }^{4} \mu \mathrm{g} / \mathrm{kg}$ of rind } \\
\hline & & (Group 2) & (Group 3) & (Group 4) & & Heavy & Light & Total \\
\hline AS5 & 66.9 & $1,155.5$ & $1,778.1$ & 146.3 & $3,146.8$ & 5.25 & 734.30 & 739.55 \\
\hline AS4 & 51.6 & 530.5 & $1,685.4$ & 112.2 & $2,379.6$ & 0.74 & 84.97 & 85.71 \\
\hline AS6 & 35.1 & 444.6 & 933.1 & 60.3 & 1,473.1 & 0.20 & 105.32 & 105.52 \\
\hline AS2 & 10.9 & 108.4 & 326.7 & 38.7 & 484.7 & 0.19 & 42.27 & 42.46 \\
\hline PP3 & 116.8 & $1,098.6$ & $1,722.9$ & 544.6 & $3,483.0$ & 1.31 & 191.80 & 193.11 \\
\hline PP4 & 26.2 & 342.8 & 292.1 & 89.5 & 750.6 & 0.28 & 28.93 & 29.21 \\
\hline PP5 & 11.0 & 271.1 & 319.5 & 34.0 & 635.6 & 0.38 & 70.37 & 70.75 \\
\hline PP2 & 27.2 & 161.8 & 225.1 & 64.2 & 478.3 & 0.14 & 52.17 & 52.31 \\
\hline
\end{tabular}

the composition of the food submitted to the smoking process, the way this process is carried out, and its intensity. Table 4 shows the concentrations of some groups of typical smoke components in the headspace of these cheeses, which are related to the smoking degree, together with the concentrations of heavy, light, and total PAH. Among the smoke components, the group of carbonyl derivatives (group 1) included furanmethanol, cyclotene, and maltol; the group of phenol and derivatives (group 2) included phenol, 2-methylphenol, 4- + 3-methylphenols, 2,6-dimethylphenol, 2,3-dimethylphenol, 2,4- + 2,5-dimethylphenols, 2,4,6-trimethylphenol, 2-ethylphenol, and 4-propylphenol; the group of guaiacol (2-methoxyphenol) and derivatives (group 3) included guaiacol, 4-methylguaiacol, 4-ethylguaiacol, 4-vinylguaiacol, 4-(2-propenyl)-guaiacol (eugenol), 4propylguaiacol, and 2 isomers of 4-(1-propenyl)-guaiacol (isoeugenol); and the group of syringol (2,6-dimethoxyphenol) and derivatives (group 4) included syringol, 4-methylsyringol, 4-ethylsyringol, and 3 isomers of 4(2-propenyl)-syringol. The influence of the vegetable matter is notable in the samples submitted to the highest degree of smoking intensity. It is evident that samples AS5 and PP3, having a similar total content of smoke components, and thus, a similar smoking degree, have different concentrations of heavy (from ben$\mathrm{z}$ (a)anthracene onwards) and light (from naphthalene to benz(a)anthracene) PAH. However, this influence is less clear in the samples submitted to a lesser degree of smoking.

Among the cheeses smoked with the same type of vegetable matter, the degree of smoking seemed to influence mainly the concentration of the heavy PAH. As Table 4 shows, decreasing values in total smoke component concentrations were associated with decreasing values in heavy PAH concentrations.

In relation to the presence of carcinogenic $\mathrm{PAH}$, there were compounds with different degrees of carcinogenicity in all the samples studied. Thus, naphthalene, recently considered as "possibly carcinogenic to humans" by the International Agency for Research on Cancer (IARC, group 2B; IARC, 2002), and benz(a)anthracene, considered as "probably carcinogenic to humans" by IARC (group 2A; IARC, 1983), were present in the outer part of all the cheeses studied. Although the level of benz(a)anthracene was very low in all cases (0.03 to $1.01 \mu \mathrm{g} / \mathrm{kg}$ ), naphthalene was the most abundant PAH in almost all cheeses. Despite naphthalene not being considered carcinogenic for many years, it was shown in 2000 that this PAH showed carcinogenic activity in rats (NTP, 2000). Consequently, international agencies such as IARC and the US Environmental Protection Agency reclassified this compound as a potential carcinogen in humans (Preuss et al., 2003). Therefore, although the determination of this compound is not usually made and no limit for its concentration in food has been suggested in international law, its presence cannot be ignored.

Other PAH with high molecular weight and considered as "possibly carcinogenic to humans" by the IARC were found in the outer part of samples AS5 and PP3 at very low concentrations. For example, benzofluoranthenes and indeno(1,2,3-cd)pyrene were present (IARC, 1983), albeit at very low concentrations ( 0.05 to 0.68 $\mu \mathrm{g} / \mathrm{kg}$ ).

Although most of the alkylated PAH, especially those derived from light $\mathrm{PAH}$, were not classified with respect to carcinogenicity, some authors have commented that 
the presence of alkyl groups can confer or intensify the carcinogenic potential of PAH (Irwin et al., 1998). However, until now, these compounds have been ignored in international food regulations.

Benzo(a)pyrene, one of the most carcinogenic PAH according to different classifications (Guillén and Sopelana, 2003), and recently included by the IARC in group 1 (carcinogen to humans; Straif et al., 2005), was identified in 2 of the samples (AS5 and PP3) at concentrations of 0.51 and $0.08 \mu \mathrm{g} / \mathrm{kg}$, respectively. These values are well within the limits in the rind of ripened smoked cheese established by Spanish law, which is $10 \mu \mathrm{g} / \mathrm{kg}$ (BOE, 1985). On the other hand, if the concentrations of benzo(a)pyrene in the cheeses studied herein are compared with those reported by other authors, it must be considered whether the data correspond to the rind of the cheeses (the most contaminated zone), or to cheeses from which the rind has been removed, or from samples of rind together with the paste. In general, the values obtained in the current study are in the same range as those found by other authors in commercial or traditionally smoked cheeses, and range from 0.04 to $7.8 \mu \mathrm{g} / \mathrm{kg}$ (Potthast, 1977; Lintas et al., 1979; Joe et al., 1984; De Martin et al., 1998; Michalski and Germuska, 2003; Pagliuca et al., 2003; Anastasio et al., 2004).

Considering the results of a previous study (Guillén and Sopelana, 2004), according to which benzo(a)pyrene was concentrated almost exclusively in the exterior part of the cheese piece and, the proportion of this part in relation to the whole cheese, benzo(a)pyrene concentrations corresponding to the whole product were calculated. Thus, considering that the percentages of the external part in relation to the whole cheese are $32.2 \%$ in AS5 and 37.1\% in PP3, the concentrations of benzo(a)pyrene per kilogram of whole cheese would be $0.16 \mu \mathrm{g} /$ $\mathrm{kg}$ in AS5 and $0.03 \mu \mathrm{g} / \mathrm{kg}$ in PP3. These values are much lower than the maximum of $5 \mu \mathrm{g} / \mathrm{kg}$ recently established by the European Union for smoked meat products and some smoked fish products (European Union, 2005), although this regulation makes no reference to smoked cheese. The concentration of benzo(a)pyrene in PP3 complied with the maximum level of $0.03 \mu \mathrm{g} / \mathrm{kg}$ permitted in food smoked with smoke flavorings (European Union, 1988), whereas in AS5 the amount of benzo(a)pyrene $(0.16 \mu \mathrm{g} / \mathrm{kg})$ was only slightly higher than this limit. However, this legal requirement does not concern cheeses treated with smoke.

\section{ACKNOWLEDGMENT}

This work was supported by the Ministerio de Ciencia y Tecnología (AGL2006-01381); CAL02-075-C3-2) and the Universidad del País Vasco (GIU05/25). G. Palencia thanks the Universidad del País Vasco for a predoctoral fellowship. The authors thank Maria Fresno, the Palmero artisans, and the Consejo Regulador de la Denominación de Origen Protegida de Queso Palmero for the cheese samples.

\section{REFERENCES}

Anastasio, A., R. Mercogliano, L. Vollano, T. Pepe, and M. L. Cortesi. 2004. Levels of benzo[a]pyrene (BaP) in "Mozzarella di Bufala Campana" cheese smoked according to different procedures. J. Agric. Food Chem. 52:4452-4455.

Badger, G. M., R. W. L. Kimber, and T. M. Spotswood. 1960. Mode of formation of 3,4-benzopyrene in human environment. Nature 187:663-665.

Bosset, J. O., U. Bütikofer, O. Dafflon, H. Koch, L. Scheurer-Simonet, and R. Sieber. 1998. Teneur en hydrocarbures aromatiques polycycliques de fromages avec et sans flaveur de fumée. Sci. Aliments 18:347-359.

Conde, F. J., J. H. Ayala, A. M. Afonso, and V. González. 2005. Polycyclic aromatic hydrocarbons in smoke used to smoke cheese produced by the combustion of rock rose (Cistus monspeliensis) and tree heather (Erica arborea) wood. J. Agric. Food Chem. 53:176-182.

Crittenden, B. D., and R. Long. 1976. The mechanism of formation of polynuclear aromatic compounds in combustion systems. Pages 209-223 in Carcinogenesis-A Comprehensive Survey. Vol. 1. R. I. Freudenthal and P. W. Jones, ed. Raven Press, New York, NY.

De Martin, S., P. Matcovich, D. Nanut, S. Zulian, and V. Siardi. 1998 Determination of polycyclic aromatic hydrocarbons (PAH) in smoked foods. Boll. Chim. Igien. Parte Sci. 49:177-181.

European Union. 1988. Council Directive 88/388/EEC of 22 June 1988 on the approximation of the laws of the Member States relating to flavorings for use in foodstuffs and to source materials for their production. Off. J. L 184:15.07.1988.

European Union. 2005. Regulation (EC) no. 208/2005 of the Commission of 4 February 2005 amending Regulation (EC) No 466/2001 as regards polycyclic aromatic hydrocarbons. Off. J. L 34:8.02.2005.

Freitas, C., and F. X. Malcata. 2000. Microbiology and biochemistry of cheeses with Appélation d'Origine Protegée and manufactured in the Iberian Peninsula from ovine and caprine milks. J. Dairy Sci. 83:584-602.

Frenklach, M., and J. Warnatz. 1987. Detailed modelling of PAH profiles in a sooting low-pressure acetylene flame. Combust. Sci. Technol. 51:265-283.

Guillén, M. D., M. L. Ibargoitia, P. Sopelana, and G. Palencia. 2004b. Components detected by headspace-solid phase microextraction in artisanal fresh goat's cheese smoked using dry prickly pear (Opuntia ficus indica). Lait 84:385-397.

Guillén, M. D., M. L. Ibargoitia, P. Sopelana, G. Palencia, and M. Fresno. 2004a. Components detected by means of solid-phase microextraction and gas chromatography/mass spectrometry in the headspace of artisan fresh goat cheese smoked by traditional methods. J. Dairy Sci. 87:284-299.

Guillén, M. D., and P. Sopelana. 2003. Polycyclic aromatic hydrocarbons in diverse foods. Pages 175-198 in Food safety: Contaminants and toxins. J. P. F. D'Mello, ed. CABI Publishing, Wallingford, UK

Guillén, M. D., and P. Sopelana. 2004. Occurrence of polycyclic aromatic hydrocarbons in smoked cheese. J. Dairy Sci. 87:556-564.

Guillén, M. D., P. Sopelana, and M. A. Partearroyo. 2000a. Determination of polycyclic aromatic hydrocarbons in commercial liquid smoke flavorings of different composition by gas chromatographymass spectrometry. J. Agric. Food Chem. 48:126-131.

Guillén, M. D., P. Sopelana, and M. A. Partearroyo. 2000b. Polycyclic aromatic hydrocarbons in liquid smoke flavorings obtained from different types of wood. Effect of storage in polyethylene flasks on their concentrations. J. Agric. Food Chem. 48:5083-5087.

International Agency for Research on Cancer (IARC). 1983. Polynuclear aromatic compounds, part 1: Chemical, environmental and experimental data. In IARC Monographs on the evaluation of 
the carcinogenic risk of chemicals to humans. Vol. 32. IARC, Lyon, France.

International Agency for Research on Cancer (IARC). 2002. Some traditional herbal medicines, some mycotoxins, naphthalene and styrene. In IARC Monographs on the evaluation of the carcinogenic risk of chemicals to humans. Vol. 82. IARC, Lyon, France.

Irwin, R. J., M. VanMouwerik, L. Stevens, M. D. Seese, and W. Basham. 1998. Environmental Contaminants Encyclopedia. National Park Service, Water Resources Division, Fort Collins, Colorado. www.nature.nps.gov/hazardssafety/toxic/

Joe, F. L., Jr., J. Salemme, and T. Fazio. 1984. Liquid chromatographic determination of trace residues of polynuclear aromatic hydrocarbons in smoked foods. J. AOAC 67:1076-1082.

Lintas, C., M. C. De Matthaeis, and F. Merli. 1979. Determination of benzo[a]pyrene in smoked, cooked and toasted food products. Food Cosmet. Toxicol. 17:325-328.

Maga, J. A. 1988. Smoke in Food Processing. CRC Press, Boca Raton, FL.

Michalski, R., and R. Germuska. 2003. The content of benzo(a)pyrene in Slovakian smoked cheese. Pol. J. Food Nutr. Sci. 12:33-37.

Möhler, K. 1980. El ahumado. Acribia, Zaragoza, Spain.

National Toxicology Programme (NTP). 2000. Toxicology and carcinogenesis studies of naphthalene (CAS No. 91-20-3) in F344/N rats (inhalation studies). NTP Technical Report Series No. 500. NIH publication No. 01-4434. US Department of Health and Human Services, NTP, Research Triangle Park, NC.
BOE (Boletín Oficial del Estado). 1985. Orden de 29 de noviembre de 1985 por la que se aprueban las normas de calidad para quesos y quesos fundidos destinados al mercado interior. B.O.E. 292, 6.12.1985. BOE, Madrid, Spain.

BOE (Boletín Oficial del Estado). 2001. Orden de 31 de agosto de 2001 por la que se ratifica el Reglamento de la Denominación de Origen "Queso Palmero" y de su Consejo Regulador. B.O.E. 128, 11.9.2001. BOE, Madrid, Spain.

Pagliuca, G., T. Gazzotti, E. Zironi, G. P. Serrazanetti, D. Mollica, and R. Rosmini. 2003. Determination of high molecular mass polycyclic aromatic hydrocarbons in a typical Italian smoked cheese by HPLC-FL. J. Agric. Food Chem. 51:5111-5115.

Panalaks, T. 1976. Determination and identification of polycyclic aromatic hydrocarbons in smoked and charcoal-broiled food products by high pressure liquid chromatography and gas chromatography. J. Environ. Sci. Health B11:299-315.

Potthast, K. 1977. Polycyclic aromatic hydrocarbons in smoked meat products. An application of a new method. Acta Aliment. Pol. 3:195-201.

Preuss, R., J. Angerer, and H. Drexler. 2003. Naphthalene-An environmental and occupational toxicant. Int. Arch. Occ. Environ. Health. 76:556-576.

Riha, W. E., W. L. Wendorff, and S. Rank. 1992. Benzo(a)pyrene content of smoked and smoked-flavored cheese products sold in Wisconsin. J. Food Prot. 55:636-638.

Straif, K., R. Baan, Y. Grosse, B. Secretan, F. El Ghissassi, and V. Cogliano. 2005. Carcinogenicity of polycyclic aromatic hydrocarbons. Lancet 6:931-932. 